\title{
The Theodorus of Cyrene Spiral Geometry and Its Application to Antenna Design
}

\author{
Anna Papió Toda, Nicolaos G. Alexopoulos, and Franco De Flaviis
}

\begin{abstract}
In this article, we introduce for the first time the radiation properties of a spiral shape that is obtained from the work on irrational numbers by Theodorus (465 BC-398 BC) of Cyrene (presently near Shahhat, Libya). In the past, this piecewise continuous spiral shape has been erroneously introduced in the literature as the Root, Pythagorean, or Einstein spiral. In addition, we use here P. J. Davis's interpolation function to obtain the continuous version of this spiral, and we present both the discrete and the continuous Theodorus spiral antenna radiation properties. Finally, we fabricate and perform measurements on the Theodorus spiral, and we present a comparison with its theoretically obtained radiation properties as well with those obtained for the standard Archimedean shape of the same diameter.
\end{abstract}

\section{Introduction}

Theodorus lived in Cyrene, an ancient city founded in $631 \mathrm{BC}$ in Libya [1]. Very little is known about him and his work other than a short discussion in Plato's Dialogues Theaetetus, Sophist, and Statesman [2]. In Theaetetus, it is mentioned that Theodorus solved the problem of irrational numbers up to the number $\sqrt{17}$. There has been extensive discussion in the literature as to why Theodorus stopped his investigation with the $\sqrt{17}$. We will provide an answer to this speculation in Section 2 of the article, where we will also introduce the interpolation function [3-5] for the continuous form of the Theodorus spiral. In Section 3, we will provide experimental data for the Theodorus spiral antenna as well as a comparison with the standard Archimedean spiral [6] of the same diameter.

\section{The Theodorus Spiral}

A description of Theodorus's work on irrational numbers leads to Figure 1, where he started with a right triangle with unit length sides to obtain the hypotenuse as the $\sqrt{2}$ (that the $\sqrt{2}$ is an irrational number was

Manuscript received 25 February 2021.

Anna Papió Toda was a PhD student at the University of California, Irvine when this work was done. She is now with Apple Corporation, One Apple Park Way, Cupertino, CA 95014; e-mail: annapapio@gmail.com

Nicolaos G. Alexopoulos is with the Broadcom Foundation, 4041 MacArthur Boulevard, Suite 510 Newport Beach, California 92660, USA; e-mail: alfios@brcmfdn.org.

Franco De Flaviis is with the Department of Electrical Engineering and Computer Science, University of California, Irvine, Room 2233, Engineering Gateway, Irvine CA 92612, USA; e-mail: franco@uci.edu.

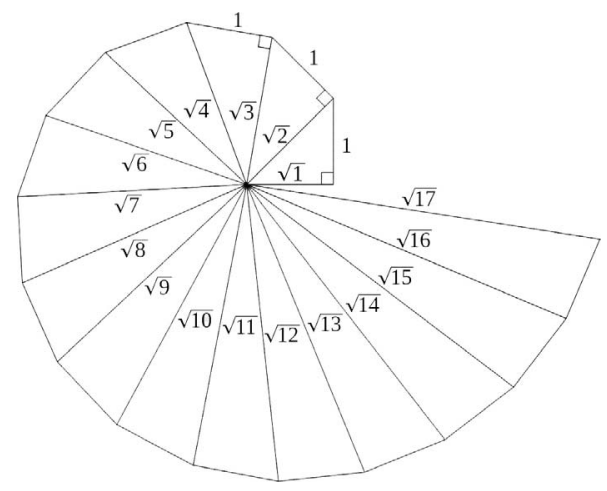

Figure 1. The discrete Theodorus spiral.

already known) and continued by erecting a perpendicular segment of unit length on the hypotenuse and so on until he reached the $\sqrt{17}$ [3-5]. The obvious question comes up as to why he stopped at the $\sqrt{17}$. The answer is that he stopped when the triangles begin to overlap [5].

This is a spiral composed of contiguous right triangles with the short side of each triangle having the value of 1 and each connected to the short side of the next triangle. A version of this spiral can also be constructed by using the equations $r(n)=\sqrt{n}+1$ and $\phi(n)=\tan ^{-1}(1 / \sqrt{n})$ with $n=1,2,3, \ldots[3,4]$ to obtain the discrete point spiral and subsequently drawing the orthogonal triangles connecting the points as shown in Figure 2. Furthermore, the discrete Theodorus spiral can be interpolated by a smooth curve using the function [3, pp. 37-38):

$$
T(x)=\prod_{k=1}^{\infty} \frac{1+i / \sqrt{k}}{1+i / \sqrt{x+k}} \quad(-1<x<\infty)
$$

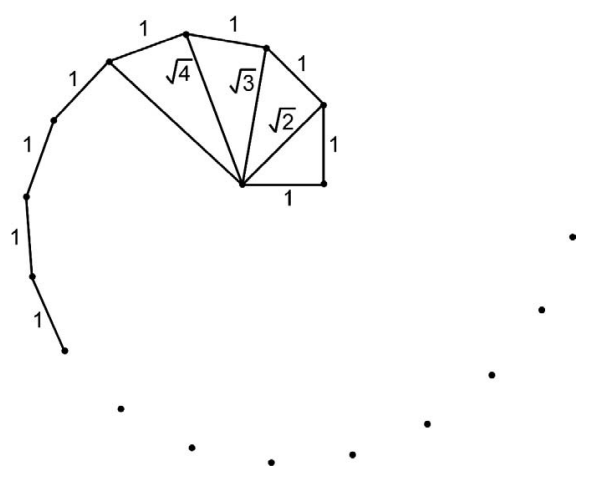

Figure 2. The discrete point Theodorus spiral. 


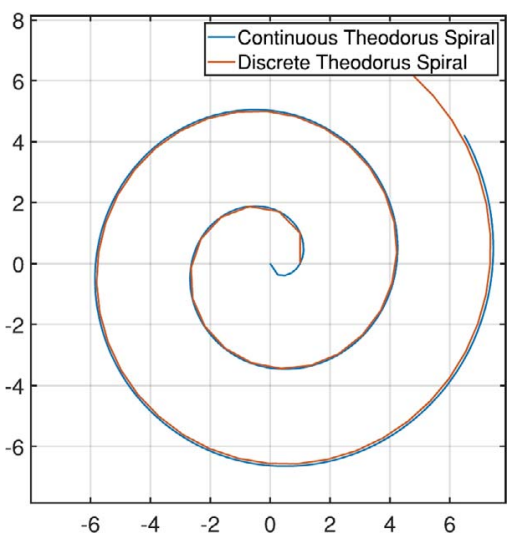

Figure 3. A comparison between discrete and continuous Theodorus spirals.

Figure 3 shows a comparison of the discrete and continuous versions of the Theodorus spiral. It can be observed that the continuous spiral follows exactly all the discrete Theodorus spiral points, with the deviation observed as the spiral grows outward due to limiting $k$ in (1) to 100,000 points. We next consider the wellknown Archimedean spiral, which is represented by the equation

$$
r=a+b \phi
$$

Figure 4 demonstrates a comparison of the two spirals for $a=0.1$ and $b=0.5$ and for $a=1.0$ and $b=$ 0.1 . Clearly, for the proper $a$ and $b$ values, the Archimedes spiral can approximate very closely the Theodorus spiral, while as the parameter $a$ increases and $b$ decreases, it contracts to a much smaller spiral.

\section{Experimental Results and Comparison With Theory}

We next study the antenna properties of both the discrete and the continuous versions of the Theodorus spiral (or equivalently an Archimedes spiral with the appropriately chosen $a$ and $b$ parameters). A picture of the Theodorus spiral (before assembly) along with its feed network is shown in Figure 5. The fabricated Archimedean spiral having the same dimensions is shown in Figure 6 along with the same feeding network.
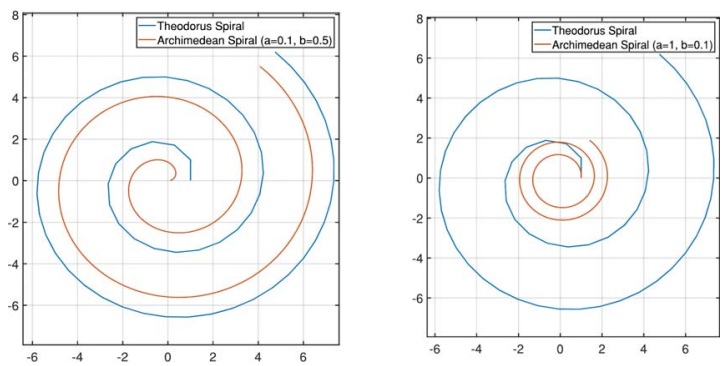

Figure 4. Comparison between the Theodorus and Archimedean spirals.
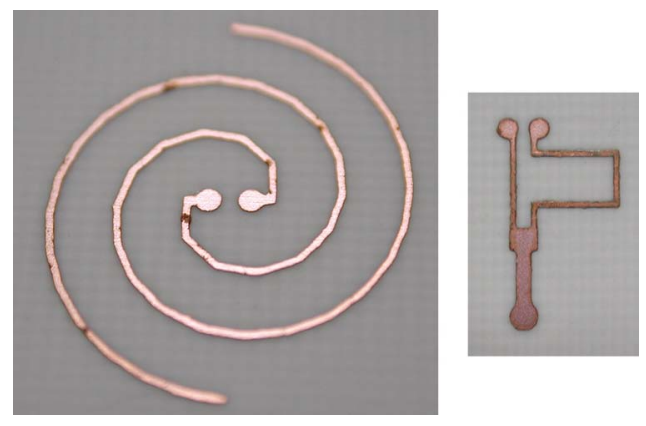

Figure 5. Fabricated discrete Theodorus spiral (left) with the corresponding feeding network (right).

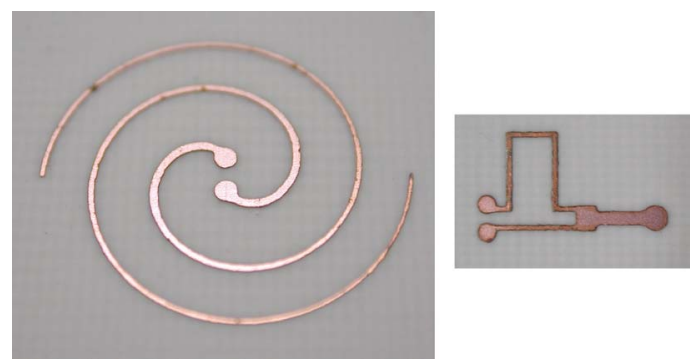

Figure 6. Fabricated discrete Archimedean spiral (left) with the corresponding feeding network (right).

The measured return loss for the Archimedean spiral (blue curve) is shown in Figure 7 and is compared with the simulated result (red curve). Excellent agreement between the two curves validates our simulation results.

Similarly, the measured return loss for the Theodorus spiral (blue curve) is shown in Figure 8 and is also compared with the simulated result (red curve). The simulated radiation pattern at the center band of $15 \mathrm{GHz}$ for the Archimedean spiral is shown in Figure 9 along with the measured data for both radiation planes (E-plane and H-plane) co-pol (RHCP) and crosspol components (LHCP). The corresponding radiation pattern at $15 \mathrm{GHz}$ for the Theodorus spiral is shown in Figure 10, and it is also compared with simulated data. Both experimental patterns are in excellent agreement

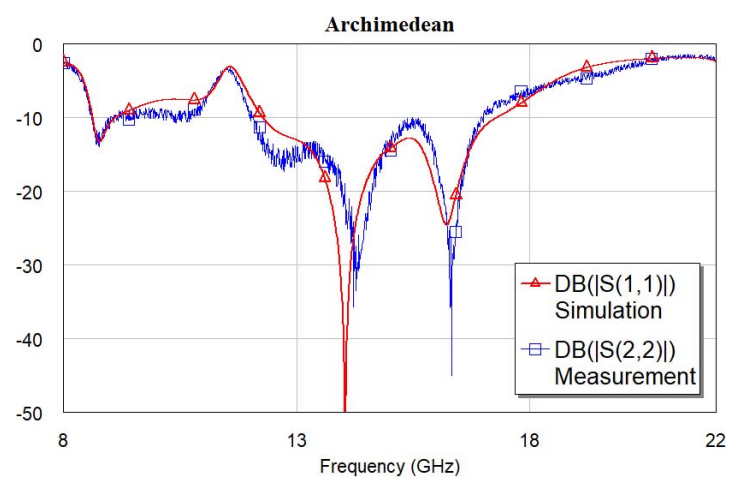

Figure 7. Archimedean spiral return loss versus frequency. Simulation (red curve) versus measurement (blue curve). 


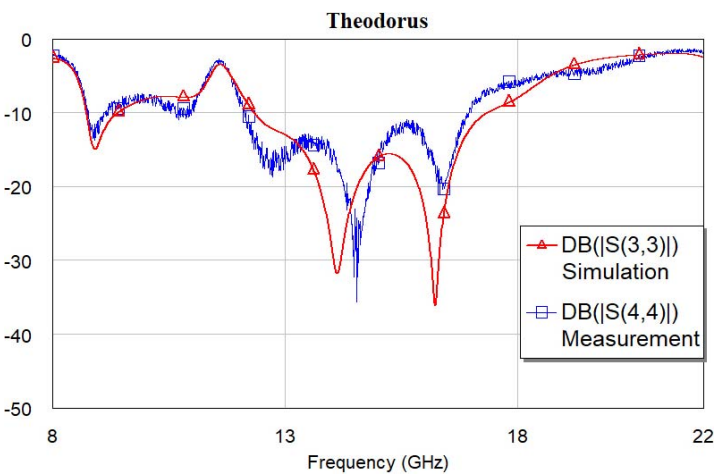

Figure 8. Theodorus spiral return loss versus frequency. Simulation (red curve) versus measurement (blue curve).

with the simulated data except for the back-radiation component, which is difficult to capture in the measurement due to the mounting post in the chamber blocking the back radiation. While both radiation patterns are similar in shape and gain on the two main planes $(\phi=0$ and $\phi=90)$, one can observe by comparing Figure 9 with Figure 10 that the Theodorus spiral exhibits a broader $3 \mathrm{~dB}$ beam width on the $\phi=0$ plane with beam width of 64 degrees versus 56 degrees for the Archimedean spiral for the RHCP component. Also, the peak gain at broadside for the Theodorus spiral is slightly higher (7.6 dBi) compared to the Archimedean spiral (7.22 dBi) by $0.4 \mathrm{dBi}$.

\section{Conclusions}

We have introduced a spiral shape attributed to Theodorus of Cyrene, Libya. Theodorus predated Archimedes by more than a century and a half. We have adopted for the first time the Theodorus discontinuous spiral shape as well as its much later developed continuous spiral form as an antenna. We have obtained its radiation properties theoretically and experimentally, and we have demonstrated that it exhibits similar

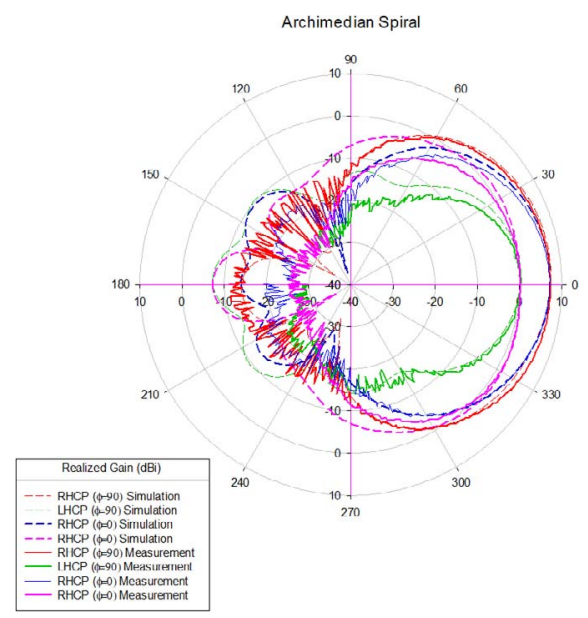

Figure 9. Comparison of simulated and measured realized gain at 15 $\mathrm{GHz}$ for the Archimedean spiral at $15 \mathrm{GHz}$.

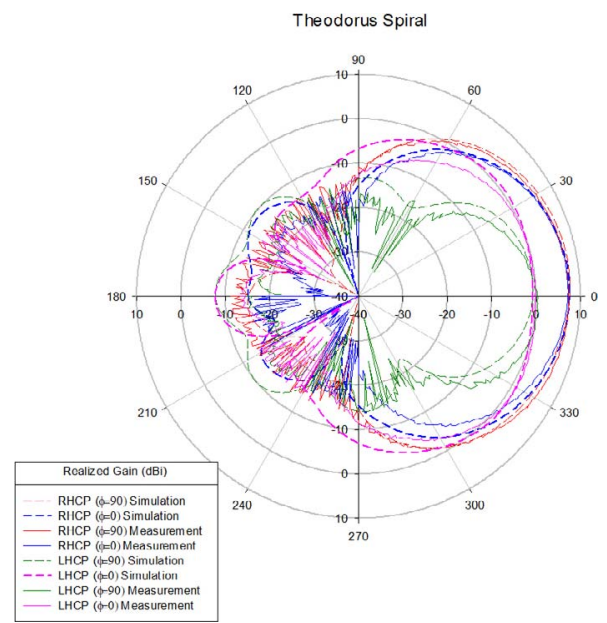

Figure 10. Comparison of simulated and measured realized gain at $15 \mathrm{GHz}$ for the Theodorus spiral at $15 \mathrm{GHz}$.

antenna properties as the Archimedean spiral but in addition provides more general design tools since it also includes the possibility for discrete spiral designs. Finally, we propose that this spiral form should be correctly referenced in the literature as the Theodorus spiral rather than the Root, Pythagorean, or Einstein spiral.

\section{References}

1. L. Campbell, The Theaetetus of Plato New York, Arno Press, 1977.

2. T. Heath, A History of Greek Mathematics, Vol. 1, New York, Dover, 1981.

3. P. J. Davis, Spirals: From Theodorus to Chaos, Wellesley, MA, A. K. Peters, 1993.

4. E. Hlawka, "Gleichverteilung und Quadratwurzelschnecke," Monatshefte für Mathematik, 89, 1980, pp. 19-44.

5. J. H. Anderhub, Aus den Papieren eines reisenden Kaufmannes, Joco-Seria, Wiesbaden, Kalle-Werke, 1941.

6. R. Netz and W. Noel, The Archimedes Codex: How a Medieval Prayer Book Is Revealing the True Genius of Antiquity's Greatest Scientist, Philadelphia, Da Capo Press, 2007. 